Mitja Skubic,

Ljubljana

\title{
IL CONGIUNTIVO ITALIANO DELLE OPERE LETTERARIE CONTEMPORANEE NELLE TRADUZIONI IN SLOVENO
}

(Ricerca di grammatica contrastiva)*

1. La grammatica contrastiva ha come scopo principale il comparare i mezzi di espressione in due o piú lingue. Tale comparazione sarà piú istruttiva per due lingue apparentate; così, per l'impiego del congiuntivo, forma verbale che qui c'interessa, le differenze constatate nell'uso in italiano $e$ in francese ${ }^{1}$ sono significative, anche se condizionate da fattori non strettamente linguistici.

La nostra ricerca si prefigge lo scopo di constatare, invece, quali siano i mezzi di espressione, in sloveno, per le varie nozioni relative a desiderio, giudizio personale, partecipazione affettiva, virtualità di un'azione, in italiano generalmente rese mediante la forma verbale chiamata congiuntivo. La ricerca è quindi ben differente da quella di un paragone nell'uso tra l'italiano e un'altra lingua romanza che possieda il congiuntivo e dove possiamo trovare concordanze e divergenze nell'uso di una stessa forma; qui si tratta di vedere se, e con quali mezzi è espressa tale nozione. Partiamo dal presupposto che la nozione sia sentita $e$ in un certo qual modo espressa anche in sloveno, sebbene non ci sia, di fronte al congiuntivo italiano, una corrispondente forma verbale.

Lo sloveno, per rendere la nozione espressa dal congiuntivo italiano, ricorre ai seguenti mezzi:

*Il presente lavoro è frutto delle discussioni nel seminario di lingua italiana presso la Facoltà di Lettere di Ljubljana negli anni 1973/74 e 1974/75, nel senso che il materiale raccolto dagli studenti veniva discusso e analizzato in classe. Così, discordando tra di noi molte volte i pareri non solo per l'analisi dell'impiego della forma verbale in italiano, ma anche di quello in sloveno, il lavoro è anche un po' il loro.

$\mathrm{E}^{\prime}$ ovvio, chi si accinge a trattare la spinosa questione dell'impiego del congiuntivo in italiano, deve tener presente i risultati delle ricerche ai quali è giunto il romanista danese Joergen Schmitt Jensen nel suo importante lavoro Subjonctif et hypotaxe en italien, Odense University Press 1970.

1 V., ad es., Christmann, Zum Verhältnis zwischen dem italienischen und dem französischen Konjunktiv. Vergleich einiger Gebrauchsweisen in Interlinguistica, Festschrift Wandruszka, Tübingen 1971. 
a) nessun segno particolare nella subordinata, dato che la congiunzione $d a$ 'che' è semanticamente svuotata; il verbo è all'indicativo

Maciste pensò che egli avesse commesso un furto e tenesse la refurtiva per le scale

Maciste je sprva pomislil, da je kaj ukradel in da je ukradeno blago pustil na stopnicah

Pratolini

La non-realtà del contenuto della subordinata è indicata dall'antecedente, dal verbo je pomislit nella principale.

b) un'altra forma verbale, e non l'indicativo, e cioè:

- condizionale

Pierretto voleva che ci andassimo noi tre

Pieretto je hotel, da $b i$ šli tja tudi mi trije

Pavese

- ottativo

Avrei dovuto ricorrere a Jane perchè ricorresse a suo padre da bom moral prositi Jane, naj pregovori svojega očeta

Soldati

- futuro

Assurdamente sperava che sua moglie non si fosse svegliata Nesmiselno je upal, da se njegova žena ne bo zbudila Berto

c) un elemento lessicale, nella subordinata con l'indicativo o aitra forma verbale, che vieta di vedere nell'azione o processo o stato una situazione precisa, e impone, invece, vedervi un'azione, uno stato, un processo generico, non rigidamente legato a una data situazione

se la frase ... detta dal Pizzuco non esprima la partecipazione e il concorso di altri

Če morda stavek... ki ga je bil izrekel Pizzuco, ne izraža morda soudeležbe tudi drugih

Sciascia

2. Il paragone così concepito ha una sua particolare importanza per la seconda lingua, per lo sloveno in questo caso, perché è in questa che si cerca di trovare $i$ vari mezzi di espressione corrispondenti per il loro significato a un'unica forma verbale in italiano. Una tale analisi, tuttavia, non potrebbe essere senza importanza nemmeno per l'italiano stesso. Prima, perché, pur lasciando da parte i passi con l'indicativo, dove si possono constatare oscillazioni o addirittura usi differenti da quella che viene considerata la norma², l'impiego di vari mezzi d'espressione in sloveno potrebbe portare a una differente valutazione del grado di virtualità di un'azione. Seconđo, perché sí

2 Alcuni usi sfuggono alla nostra analisi, ad es.: - Pensai ch'era Rosalba, che la storia non fosse finita, Pavese; vale a dire, non si z saputa trovare una ragione plausibile per l'uso, rispettivamente, dell'indicativo e del congiuntivo in una situazione sintatticamente e semanticamente identica. Un caso analogo è stato notato già đa Christmann, Zum Verhältnis ..., cit.: - Ella pensa che sia sufficiente vedere come Mario e Gesuina si tengano a braccetto e come lui è infastidito e come lei è felice, Pratolini. 
cercherà di circoscrivere l'impiego del congiuntivo italiano in tre grandi sfere, senza tuttavia delimitarle precisamente. Il valore della forma verbale rısulterà, così, più chiaro e il grado della potenzialità più evidente.

3. La nostra analisi è lungi dal mirare a offrire un quadro completo. Soprattutto, ogni "corpus« è una scelta arbitraria: questo, non solo resta limitato alla letteratura contemporanea, vale a dire alle opere apparse, con qualche rara eccezione, nel secondo dopoguerra, ma è anche condizionato da un fatto ovvio, vogliamo dire dalle traduzioni delle opere letterarie italiane in sloveno. ${ }^{3}$

Inoltre, sfuggono alla nostra analisi parecchi passi. Ne sono esclusi quei passi in cui la traduzione strutturalmente si scosta dall'originale. Il traduttore ideale, ce lo auguriamo, non traduce parole e strutture e ha tutto il diritto di eliminare una subordinata; cosi

Fossi in te non sarei tanto sicuro

$\mathrm{Na}$ tvojem mestu ne bi stal na tako tranih tleh

Pratolini

Non possiamo prendere in considerazione neanche passi in cui la forma verbale non sia decisamente un congiuntivo, quali a me basta che andiamo; basta che ti sbrighi. Solo per analogia con passi di contenuto simile, non per la forma in sé giudichiamo tale forma un congiuntivo.

Non sono stati presi in esame neanche quei passi di cui il traduttore non abbia capito bene il senso, magari conservando con una certa qual fedeltà ia forma. Sono significativi i passi con dei verbi modali quando questi diventano veri e propri ausiliari, ad es.:

Chi vorresti ti bussasse alla porta?

Koga pa bi želela, da bi potrkal na tvoja vrata?

Calvino

$E^{\prime}$ da tenere presente che abbiamo a che fare con dei testi letterari, $i$ quali, necessariamente, rispettano la norma imposta dalle grammatiche, dall'

3 Sono state prese in esame e paragonate con le rispettive traduzioni in sloveno, le seguenti opere:

Berto, Il cielo è rosso

Calvino, Il sentiero dei nidi di ragno

Cassola, La ragazza di Bube

Levi, Cristo si è fermato a Eboli

Moravia, Amor coniugale

Il disprezzo

Pavese, Il diavolo sulla collina

La bella estate

Piovene, Le Furie

Pratolini, Cronache ai poveri amanti Metello

Sciascia, Il giorno della civetta

Soldati, Lettere da Capri

Tobino, Clandestino

Il deserto della Libia

Vittorini, Conversazione in Sicilia 
uso colto. Anche qui, tuttavia, constatiamo che il maggior numero di indicativi, laddove potremmo aspettarci il congiuntivo, ossia, la più forte oscillazione nella scelta dell'una o dell'altra forma si trova nella sfera che chiamiamo del giudizio personale. ${ }^{4}$

4. La nostra attenzione è concentrata sulla proposizione formalmente dipendente. Il vero contenuto, infatti, sta proprio lì. La principale non esprime altro che il mio rapporto, il mio angolo visuale rispetto al contenuto della (formalmente) subordinata; questo, almeno per la sfera volitiva e quella dell'opinione personale. Nella terza grande sfera, quella della potenzialità, non è la principale, o il contenuto della principale che detta l'impiego del congiuntivo nella subordinata: la vera ragione di tale impiego, e dell'oscillazione nell'uso dell'uno o dell'altro modo sta nella subordinata stessa. La scelta della forma verbale, dell'indicativo o del congiuntivo è distintiva, mentre non lo è nelle prime due sfere. Nella sfera della potenzialità, in generale, l'indicativo serve ad esprimere un'azione o un processo o uno stato isolato. unico, determinato, e il congiuntivo un'azione, stato, processo generico, non specificato.

Inoltre di constatare quale mezzo sia stato scelto nella traduzione, l'analisi, anche se parziale, dell'uso del congiuntivo nell'italiano, permette di vedere se un dato mezzo linguistico in sloveno corrisponda, e sempre, ad un impiego semantico del congiuntivo italiano.

Vediamo l'impiego del congiuntivo italiano condizionato da tre nozioni, quella della volontà, quella del giudizio personale, soggettivo, e quella delia potenzialità. Ogni sistemazione, ovviamente, pecca di forzatura, giacché molte volte siamo indecisi a quale delle tre grandi categorie attribuire l'uso di un congiuntivo. Nessun'azione espressa col congiuntivo è reale: è non-reale, magari realizzabile, è virtuale. Azione o processo o stato, espressi col congiuntivo sono tutti virtuali, cioè non realizzati; solo che nelle prime due categorie (sfera volitiva, sfera del giudizio personale) tale non-realtà contiene un marchio soggettivo, e perciò non esiste scelta nè oscillazione, esiste solo, nella lingua parlata, dapprima, una lenta sparizione dell'uso della forma, mentre esiste la possibilità di sfumature nella sfera potenziale.

Avendo visto, nei passi letterari, che lo sloveno per esprimere ciò che in italiano è espresso dal congiuntivo può ricorrere a vari mezzi grammaticali o lessicali, la nostra attenzione viene concentrata su questi quesiti:

a) se, cioè, il condizionale sloveno appaia solo quando abbiamo a constatare nell'italiano la nozione della potenzialità, e se, in tale sfera, appaia sempre;

b) se, inoltre, le due lingue distinguano nettamente tra un'azione, un processo, uno stato precisati e una nozione generica, e se il mezzo linguistico che nello sloveno corrisponde al congiuntivo risulti sempre lo stesso;

4 Si veda l'acuta osservazione di Joergen Schmitt Jensen, op. cit., a pag. 343, sull' impiego, nelle dichiarative, dell'indicativo, forma più frequente del congiuntivo, in "dialogue vivant et souvent argotique du roman 'Ferito a morte' de $L a$ Capriau.

E' noto che nella lingua parlata l'uso del congiuntivo scarseggia soprattutto nelle subordinate dichiarative. 
c) se una tale genericità che appare particolarmente accentuata con una principale negativa, anche in sloveno venga in qualche modo, eventualmente sempre nello stesso modo, contrassegnata come potenziale;

d) se lo sloveno, privo di una forma verbale corrispondente al congiuntivo italiano, sfrutti vari mezzi grammaticali e lessicali in tutte e tre le sfere, calle quali partiamo, più o meno in egual misura, oppure se nella sfera volitiva $e$ in quella del giudizio personale tale procedimento sia molto meno frequente che nella sfera della potenzialita.

5. La sfera volitiva.

a) Al congiuntivo italiano corrisponde il semplice indicativo in sloveno: il verbo nella principale, tuttavia, colloca l'enunciato della subordinata nella sfera del desiderio, della finalità:

Vogliamo che le tariffe vengano rispettate

Hočemo, da upoštevate tarife

Pratolini

Io leggevo subito ... le cartoline, e gliele restituivo senz'altro, perché le mandasse alla questura

Razglednice sem prebral ... takoj in sem mu jih takoj vračal, da jih pošlje na kvesturo Levi

b) Al congiuntivo italiano corrisponde una forma verbale, che non è l'indicativo, e cioè:

a) il condizionale:

Pin ora vorrebbe che Comitato arrivasse... entrasse... e dicesse bi rekel

Zdaj bi Pin želel, da bi prišel Komite... da bi stopil... in altri

Gli insegnava a leggere e a scrivere perchè fosse più avanti degli

Učila ga je brati in pisati, da bi bil ... pred drugimi

Piovene

allora gli italiani cominciarono a desiderare che questa situazione trovasse in qualsiasi modo una via položaja

so si Italijani zaželeli, da bi se kakorkoli našel izhod iz tega

Pieretto voleva che ci andassimo

Pieretto je hotel, da $b i$ šli tja tudi mi trije

Tobino

Pavese

e dava coi calcagni nudi dei rapidi e continui colpi sui fianchi dell' asinello perchè continuasse la corsa

z naglimi in nenehnimi sunki z golimi petami $\mathrm{v}$ boke je spodbadal oslička, da bi jo odnašal naprej

Tobino

$\beta$ ) il futuro:

Vado a dirle qualche cosa perchè non stia in pensiero Grem in ji rečem, da ne bo v skrbeh

Berto

Ti ho avvertito perchè tu sappia come dobbiamo comportarci Opozorila sem te, da boš vedel, kako se morava vesti 
Speriamo che non torni in sè prima che abbia finito Upajmo, da se ne bo prej osvestil

Pratolini

Posso dire al calzolaio che venga ad aiutarti Lahko rečem čevljarju, da ti pride pomagat

Berto

Le azioni o gli stati espressi nelle subordinate ora citate sono posteriori rispetto alle azioni o stati delle principali. In italiano potrebbe esser usato il futuro; la nozione di finalità, o anche della consecutività finale, tuttavia, impone il modo congiuntivo; in sloveno è di regola usato il futuro; è possibile ricorrere al presente, solo per i verbi perfettivi. Col presente il desiderio è espresso più chiaramente; col futuro, ovviamente, la conseguenza.

$\gamma$ ) l'ottativo

avrei dovuto ricorrere a Jane perchè ricorresse a suo padre da bom moral prositi Jane, naj pregovori svojega očeta

Soldati

Disse che lo lasciassero parlare

Rekel je, da naj ga pustijo govoriti Sciascia

Le andò a dire che stesse calma

in rekel, naj se ne vanemirja Pratolini

Ti venisse 5 un cancro all'anima

$N a j$ ti rak razje dušo

Calvino

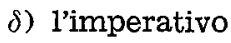

Possiamo aggiungere pochi passi in cui il congiuntivo viene tradotto con l'imperativo. Si tratta anche per l'italiano di un imperativo che però ricorre alle forme del congiuntivo; il comando viene così attenuato di molto, tutt'al più possiamo parlare di esortazione o adđirittura di desiderio.

$\begin{array}{cc}\begin{array}{ll}\text { Non mi prenda a noia } \\ \text { Ne zamerite }\end{array} & \text { Pratolini } \\ \text { Morissi } & \\ \text { Crkni } & \text { Calvino } \\ \text { Mi ascolti, avvocato } & \\ \text { Posiušajte me } & \text { Cassola }\end{array}$

c) In questa sfera non abbiamo trovato esempi da cui risulti che lo sloveno può ricorrere, nella subordinata, a un elemento lessicale, (mentre il modo verbale è quello indicativo), per indicare che l'azione della subordinata è voluta, desiderata e non realmente avvenuta.

6. Sfera del giudizio personale

Nella sfera del giudizio personale comprendiamo oltre ai passi dove è espresso un parere soggettivo, un'opinione, anche quelli dove è visibile la partecipazione affettiva, dove viene espresso lo stato d'animo.

a) Nella stragrande maggioranza dei casi lo sloveno non ha elementi, salvo il semantema del verbo della principale, per sottolineare che la realizzione

5 Crediamo inutile fare una distinzione tra l'uso nelle indipendenti e nelle subordinate, giacché esso è condizionato dagli stessi motivi. 
del contenuto nella dipendente è invalidata, perché vista attraverso il mio giudizio o attraverso la mia visione affettiva. Basterà citare pochi passi:

sei proprio sicuro che tedeschi o fascisti non sospettino nulla? ali si popolnoma gotov, da Nemci ali fašisti ničesar ne sumijo

Tobino

Non metterti in testa che gli sbirri siano tutti stupidi

Ne vtepaj si v glavo, da so vsi biriči bedaki Sciascia

in specie di una che era giunta notizia avesse gli occhi neri, fosse snella e ridesse timorosa e ladra di curiosità

o kateri so zvedeli, da je črnooka, vitka in da se smeje plaho ter da je čez vsako mero radovedna

Tobino

Anche lui parlava molto di onore... ma noi sappiamo oggi chi fosse Hitler e che cosa fosse il suo onore 6

Tudi on je veliko govoril o časti... toda mi danes vemo, kdo je bil Hitler in kakšna je bila njegova cast

Moravia

Capisco ora cosa sia un pezzente

Sedaj vem, kaj je razcapanec

Pavese

è meglio che me ne vaäa

je bolje, da pri priči odidem

Pratolini

lamentò che non fosse stato fatto un sopraluogo

pritožil se je nad tem, da niso opravili ogleda na kraju samem

Cassola

D'altra parte non mi dispiaceva che non venisse

Po drugi plati pa sem bil kar zadovoljen, da ne gre z menoj

Moravia

Non mi dispiacque che la cosa finisse li

Mi je bilo čisto prav, da se je stvar tako končala

Pavese

b) Il congiuntivo dell'originale viene trađotto con una forma verbale che include in sé l'idea della non-realtà, e più precisamente

a) col condizionale:

e aveva paura che il prete si voltasse e lo riconoscesse

Bube se je bal, da bi se duhornik obrnil in ga spoznal

Cassola

Io, che si possa vincere con la scheda, ci ho sempre creduto poco nicami

Zmeraj sem bolj malo verjel, da bi lahko zmagali z glasov-

6 Un congiuntivo piuttosto insolito per la normatività italiana. Abbiamo a che fare col discorso indiretto e, benché il verbo sapere, affermativo, sembri assicurare la realta dello stato espresso nella subordinata, è ben chiaro che si tratta di un'opinione personale. Non è un'azione o stato potenziale; un legame con la potenzialità esiste, tuttavia, determinatezza dell'azione è attenuata, offuscata, come lo è nella sfera potenziale.

Passi di tale tipo saranno sconosciuti, credo, alla lingua parlata, e scarsi anche nella lingua letteraria. Citerei ancora un passo da Bacchelli, Il mulino del Po, II, pag. 200: - Segui poi la fuga di Sua Santità a Gaeta e la Costituente, che ben sapete che cosa fosse. 
cosí non si corre pericolo immediato che arrivi e lagli l'acqua vodo

in tako zaenkrat ni nobene nevarnosti, da bi prišel in zaprl

Suppongo che tale giudizio ... fosse giusto in complesso

Zdi se mi, da bi bila taka sodba... na splos̆no pravilna

Moravia

Io non dico che lei, dal suo punto di vista, non possa anche aver ragione

Ne pravim, da s svojega stališča tudi vi ne bi imeli prav

Pratolini

non ebbe bisogno che il maresciallo facesse da interprete

kapetanu ni bilo treba, da bi mu marescialo prevajal

Sciascia

p) col futuro, solo quando l'azione è posteriore rispetto all'azione o stato o processo della reggente. Il che vuol dire che, a rigor di consecutio temporum, ci si aspetterebbe un condizionale (o condizionale composto, piuttosto), ma che la necessità di esprimere la nozione dell'opinione personale sopraffà quella di concordare i tempi. Storicamente, il problema non si pone nemmeno, essendo il condizionale un composto con l'imperfetto nella maggior parte delle lingue romanze: l'impiego del congiuntivo dell'imperfetto è dunque da considerarsi regolare anche in una lingua, quale appunto l'italiano, che per la composizione ricorre al perfectum del verbo habere, giacché il congiuntivo del perfectum deve esser sparito da tempo. Lo sloveno conosce in questo caso il futuro, la consecutio temporum alla latina non vi è, infatti, seguita.

Così troviamo nelle traduzioni il futuro oppure anche il presente, sempre di un verbo perfettivo, col valore e in funzione di un futuro.

gli dispiaceva sfuggisse dalle loro mani žal mu je bilo, da jim uide iz rok

Tobino

Aveva il terrore che mi facessi prete Bal se je, da bom tudi jaz duhovnik

Pavese

tutti avevano firmato non credendo che venisse la guerra $\checkmark$ prepričanju, da vojske ne bo, so vsi podpisali

Tobino

$\gamma$ ) Nella sfera del giudizio personale o della partecipazione affettiva, nelle traduzioni non appare l'ottativo; è comprensibile, giacché l'ottativo è legato strettamente all'espressione della volontà, del desiderio.

c) Nella sfera del giudizio personale, la congiunzione in sloveno può essere kot da 'come se', la quale rispetto al semanticamente svuotato da 'che', esprime la nozione del pensato e non un'azione o stato reali. E' la stessa che serve nelle subordinate comparative irreali, anche lì, ovviamente, per esprimere un paragone irreale.

Pareva avesse preparato un discorso e ora gli venissero a mancare le parole

Zdelo se je, kot da je pripravil cel govor

Pratolini 
era sembrato al tenente, mentre l'arabo passava vicino, che questo fosse come spinoso 7

je trnov

ko je šel Arabec mimo njega, se je poročniku zazdelo, kot da

semprava che avesse in animo di frustrarli vse je kazalo, kot da jih bo pretepal

Tobino

Tobino

Altro elemento lessicale che invalida la realtà della dichiarazione può essere anche un avverbio, quale verjetno 'probabilmente':

Là dove è da credere che l'intreccio si facesse più appassionato e più pauroso, notai che...

In opazil sem, da se ji je na tistih mestih, kjer je bila zgodba verjetno najbolj napeta...

Moravia

Interessa soprattutto l'italiano la scelta del modo nella subordinata esprimente un'opinione soggettiva con, nella reggente, un superlativo o un'espressione superlativa. E' raro trovare l'indicativo; cosi in Pratolini: "E' la prima fotografia che mi faccio ..... In sloveno appare sempre l'indicativo e quasi sempre accompagnato da un elemento generalizzante:

il più grande parlatore che avesse mai incontrato največji govornik, kar jih je bil kdaj srečal

Sciascia

ed era quello l'unico luogo nel quartiere dove apparisse il cielo s katerega si lahko videl vsaj nekaj neba

Berto

Era ben raro che una bestia gli morisse dopo l'operazione prav redko se je primerilo, da mu je po operaciji kakšna žival poginila

Levi

7. Sfera della potenzialità

La sfera della potenzialità è quella più ampia, e anche quella meno palpabile. Vogliamo dire che il congiuntivo italiano, nei suoi valori che crediamo poter riunire sotto il nome di spotenziali", serve ad esprimere una grande varietà di nozioni; d'altra parte, non tutto ciò che è potenziale viene espresso per mezzo del congiuntivo. E' però senz'altro vero che la potenzialità viene rafforzata dall'impiego del congiuntivo, ad es. nella protasi del periodo ipotetico. 8

E' legata strettamente alla potenzialità di un' azione la nozione della sua genericità. 9 Tale genericità può esser annunciata nella reggente da un antecedente, quale un pronome indefinito, un articolo indefinito (o la mancanza dell'articolo); tale elemento di genericità, tuttavia, può trovarsi nella dipendente stessa. Un gran numero di questa nozione della sfera potenziale sono

7 Significativo l'impiego di come nell'originale italiano.

8 Cfr. il profondo studio sul Congiuntivo potenziale nell'antico italiano di Franca Brambilla Ageno in Il verbo nell'italiano antico, Milano-Napoli 1964, p. 334 ss.

9 Gili y Gaya, Curso superior de sintaxis española, Barcelona 1970, pag. 136, condensa il suo pensiero così: - En las oraciones de relativo se pone el verbo en indicativo cuando el antecedente es conocido; si es desconocido o dudoso, el verbo va en subjuntivo: Haré lo que usted manca (presente; el mandato es conocido) / Haré lo que usted mande (futuro; cumpliré la parte conocida y desconocida de su mandato). 
periodi che nella reggente contengono una negazione, del tipo »Ma non erano cose che avessero importanza« (Berto).

Con una reggente negativa è difficile immaginare l'indicativo nella subordinata; vorremmo, tuttavia, asserire che l'importante è di avere un antecedente, un elemento generalizzante nella reggente; e una negazione lo è, giacché si oppone, in una data situazione, a vedere la realizzazione di un'unica azione. Tutte le grammatiche normative danno esempi analoghi al passo

$$
\begin{gathered}
\text { cerchiamo ... qualcosa che rompa o svari la giornata } \\
\text { nekaj, da bi bil dan zanimivejši in pestrejši }
\end{gathered}
$$

Pavese

Del resto, non è solo la negazione elemento di genericità; lo è anche l'ínterrogazione. Né in una negativa né in una interrogativa (pensiamo, certo, al periodo e non a una sola proposizione) l'azione, lo stato o il processo sono presentati come realmente accaduti o esistenti. ${ }^{10}$ Se è vero che è la reggente, vale a dire, formalmente la principale, che detta la scelta del modo verbale come in

e non piú nessuno in particolare che gli occupi il cuore in ni nifkogar več, ki bi imel prostor $\mathrm{v}$ tvojem srcu

Vittorini

è anche vero che il contenuto reale sta nella subordinata. L'impiego del modo condizionale nello sloveno è prezioso per constatare la potenzialità. Troviamo, tuttavia, l'indicativo, benché il condizionale non stonasse per niente, in qualche subordinata interrogativa indiretta, ad es.:

Il mattino dopo mi alzai presto e, senza indagare dove fossero Battista ed Emilia, uscii

Naslednje jutro sem vstal zgodaj in ne da bi poizvedoval, kje sta Battista in Emilija, odšel Moravia

7.1 Raggruppiamo come primi quei passi che potremmo chiamare formalmente srelativi« perché introdotti da una congiunzione o un avverbio relativi.

Formalmente troviamo parecchi passi in cui la traduzione slovena non mostra per niente che abbiamo a che fare con un'azione potenziale; tuttavia, l'antecedente è di regola imprecisato: ora, mancando nello sloveno l'articolo (determinativo e, nella lingua letteraria, anche quello indeterminativo), solo la situazione, vale a dire il contesto può precisare l'azione. Per noi, tali casi sono da annoverare tra quelli in cui figura un elemento lessicale che colloca un passo nela sfera potenziale. La sola spartizione che s'imponga per il paragone con la traduzione è quella tra l'impiego, nello sloveno, dell'indicativo o del condizionale.

a) Troviamo spesse volte l'indicativo:

I piu li credevano agenti di questura che stessero inseguendo un borsaiolo

10 Per ricorrere ancora una volta all'opera dell'insigne linguista spagnolo, ib.: - La afirmacion Hay alguien que se atreve a saltar exige indicativo; si decimos Hay alguien que se atreva a saltar insinuamos una leve duda, la cual pasará a ser más completa si se trata de una pregunta: Hay alguien que se atreva a saltar? 
Večina ljudi je mislila, da sta policijska agenta, ki zasledujeta kakega žeparja

... bastava a farle fare smorfia; oppure un bambino che correndo lungo il fossato rischiasse di caderci

... spako pa je lahko izzval tudi kak otrok, ki je tekel ob nevarni, glaboki grapi Moravia

Feci questa scoperta nei primi giorni di matrimonio e per momento ebbi quasi il senso di essere stato ingannato, come chi, essendosi sposato per tornaconto, scopra dopo le nozze che la moglie è povera ... in po poroki ugotovi, da je žena pravzaprav revna

Moravia

ho soprattutto bisogno di articoli di guarnizione, cose minute, che vadano bene sui cappelli e sui vestiti

drobnarije, ki jih lahko uporabljajo na klobukih in ablekah Pratolini

dando a tutto viso l'aspetto ripugnante di una maschera grottesca in cui a bella posta ... fossero stati appunto esagerati fino alla caricatura certi tratti

smešne krinke, na kateri so bile ... nalašc̆ še posebej poudarjene nekatere poteze

Moravia

Sembrava un bambino impaurito che stesse per piangere Podoben je bil prestrašenemu otroku, ki mu gre na jok

Pavese

Anche qui conviene sottolineare che un tale costrutto della frase è decisamente letterario. Non sorprende il numero piuttosto alto delle proposizioni di questo tipo in Moravia. Da notare l'impiego dell'avverbio lahko: accompagnando il verbo esprime quello che in italiano il verbo potere più infinito; è espressa, cioè, la potenzialità dell'azione. La traduzione slovena è perciò impeccabile.

b) Il condizionale appare in sloveno ogni volta che abbiamo un elemento negativo, sia nella reggente sia nella dipendente.

la burocrazia li indicava responsabili di tutto ciò che non fosse prono, regolare

birokracija je nalagala višjim častnikom izključno odgovornost za vse, kar bi bilo neurejenega, nepravilnega Tobino

quella forza .... che la rendeva calma e sicura di sé e indifferente a ogni cosa, che non fosse l'adempimento del suo dovere

postala je mirna in samozavestna do vsega, kar ne bi pomenilo izpolnitve njene dolžnosti

Cassola

Non ricordo che mai alcuna mia parola e alcun mio gesto provocasse in lei quella strana transformazione

ne spomnim se, da bi kadarkoli kaka moja beseda ali kretnja sprožila

Moravia

i fattori ... sanno che a Firenze non c'è maniscalco che lo valga upravniki posestev ... vsi vedo, da $v$ Florenci ni kovača, ki bi ga prekosil

Pratolini

Non avevi nessuno che ti guidasse che ti stesse vicino

Nikogar nisi imel, da bi te vodil, da bi ti stal ob strani

Cassola 
7.2 La seconda categoria del congiuntivo potenziale è quella delle modali. Il modo congiuntivo nell'imperfetto sottolinea l'irrealtà del secondo termine del paragone. Una sottocategoria delle modali potrebbe essere quella in cui il paragone è quantitativo (del tipo: .. più di quanto non si creda, e sim.). Questo tipo viene tradotto in sloveno generalmente coll'indicativo, mentre il primo conosce anche un cospicuo numero di condizionali. Certo è che già l'introduzione per mezzo di una congiunzione modale, cosi l'italiano come se, come lo sloveno kot da, kakor da, kakor če semanticamente non è neutrale: annuncia un paragone immaginario, non corrispondente alla realtà.

Sono parecchie le traduzioni contenenti l'indicativo. Cosi, ad es:

Nella piazza comparve un frate, di maniere composte, come non fosse in quel posto

$\mathrm{Na}$ trgu se je pokazal menih in se dostojanstveno držal, kot da ni na tem mestu

Tobino

Il vecchio di nuovo con fatica, come avesse una pena disse

Starec je rekel zopet $\mathrm{s}$ težavo, kakor da mu je mučno

Tobino

Era come se il sole e il peso della corrente mi avessero intriso vrlino

Bilo je, kot da sta mi sonce in težki tok vcepila neko svojo

Più frequenti sono, tuttavia, i passi con il condizionale. Si trovano persino dei passi ibridi, senza che si possa constatare una sostanziale differenza nel valore dell'una o dell'altra forma verbale. Cosí:

Con Amelia era tutto piú facile, e ci si divertiva di gusto come se niente importasse e quella sera dovessero succedere le cose piú varie

$\mathrm{Z}$ Amelijo je bilo vse laže, $\mathbf{z}$ njo se je človek lahko zabaval, kot da ni na svetu nič važnejšega, ko da bi se moraio ta večer zgoditi kdo ve kaj

Favese

L'irrealtà è, ovviamente, espressa con maggior rilievo per mezzo del condizionale. $\mathrm{Da}$ qui, la frequenza del suo impiego.

Carla ... tentò di passare, come se non li avesse visti Karla je ... poskušala iti mimo, ko da ju ne bi videla

Berto

quasi che non fossero fatte per gli uomini skoraj tak, kakor da bi ne bil ustvarjen za ljudi Berto

come se fossero cose da mangiare kakor če bi se dale pojesti

Vittorini

Anselmo senza voltarsi, si avviò spedito come dovesse raggiungere in fretta chi l'aspettava

Ne da bi se ozrl, se je Anselmo hitro napotil kot bi moral brž priti do koga, ki ga pričakuje

Tobino

quasi si studiassero per sapere chi dei due ... kot bi ugibala, kateri od njiju

Tobino

come se si trattasse di due ritrovati comuni da acquistarsi in qualsiasi farmacia

kot da bi šlo za najbolj navadni zdravili, ki bi ju lahko dobil Moravia 
ma i fatti del '79 li conosco, come se ci fossi stato

a dogodke 79. leta poznam, ko da bi bil zraven Pratolini

Sono vere subordinate modali, e senza paragone, anche quelle di sottrazione $^{11}$ : se nell'italiano la scelta del tempo non influisce sul grado della potenzialità, nelle traduzioni slovene troviamo sempre il condizionale:

E la sua mente andava, senza che egli la controlasse o ia seguisse, nella sua strada

$\mathrm{Njegov}$ duh je odhajal, ne da bi ga nadzoroval ali mu sledil po njegovi poti

Berto

e se l'operazione era riuscita senza che la busta si rompesse o ne restassero tracce...

in če se je operacija posrečila, ne da bi se raztrgal ovoj ali da $b i$ ostali sledovi

Levi conto

II suo istinto originale riaffiorava - e senza che ella se ne rendesse

Njena resnična nrav se je spet zganila in - ne da bi se tega zavedala

Pratolini

7.3 Il congiuntivo appare in italiano nelle subordinate che esprimono un' azione o stato o processo potenziali che possiamo in qualche modo riunire nella stessa nozione del nesso causale. Rientrano in tale nozione le autentiche subordinate causali (per l'impiego del congiuntivo interessano, ovviamente, solo quelle dove si nega l'effetto di una causa), condizionali e concessive.

7.3.1. $\mathrm{E}$ ' negato l'effetto della causa (icause brisée«) e l'italiano ricorre al congiuntivo "per caratterizzare una causa irreale ${ }^{12}$. Lo sloveno accentua l'irrealtà, o meglio, la potenzialità che si dichiara negata con un elemento negativo nella reggente, servendosi del condizionale:

e non perché quella gente sapesse di mitologia, ma per via del gruppo del Bandinelli logijo

a ne zato, ker bi se ljuđje iz te četrti kaj spoznali na mito-

Non è che abbia proprio paura, sai

Saj ne, da bi me bilo strah, veš

Pratolini

Berto

7.3.2 Le suburdinate condizionali possono formare il periodo ipotetico e conoscono, in italiano, il congiuntivo imperfetto o piuccheperfetto nella protasi, a seconda che la condizione sia presentata come (formalmente) potenziale, vale a dire realizzabile, oppure come irreale; nell'apodosi, l'italiano ricorre al condizionale. Lo sloveno conosce il condizionale in ambedue le parti.

Se si dovesse trattare così le canaglie, non resterebbe una sola persona al mondo

Ko bi morali z lopovi tako ravnati, ne bi ostal na svetu niti en človek

Piovene

11 E' l'espressione usata dal Tekavčič, Grammatica storica dell'italiano, II, Bologna 1972, par. 1276. Sembra più riuscita di 'eccettuativa'.

12 Regula-Jernej, Grammatica italiana descrittiva, Bern 1965, pag. 282. 
Capirei se copiassero un uomo

Razumela bi, ko bi risale moškega

Pavese

Se ci si mettesse d'accordo sarebbe bello Ce hi se lahko pobotal z njim, bi bilo dobro Calvino

pensa come sarebbe stata felice se lo avesse conosciuto prima ... kaǩo bi bila srečna, če bi ga spoznala prej Pratolini

del denaro che si sarebbe perso se l'R.T non avesse piú potuto comunicare con la radio denarja, ob katerega bi bili, če bi radiotelegrafist, ne mogel poročati po radiu

Tobino

E' ovvio che l'ipotesi può anche non essere incatenata cosí rigidamente al formale periodo ipotetico. In un certo qual senso, anche una subordinata condizionale esprime un fatto generico, non punta su un'azione ben precisa; cosí possiamo giustificare l'impiego del congiuntivo sia come esprimente la genericità, sia come protasi di un periodo ipotetico in passi come "Uno che facesse tutti i giorni questa vita, diventerebbe animale - "Tisti, ki bi ves dan takole živel, bi postal živalı (Pavese).

Nelle subordinate condizionali isolate, non legate nel periodo ipotetico, lo sloveno ricorre per lo piú all'indicativo accompagnato da un elemento lessicale che piú fortemente della sola congiunzione condizionale če 'se' segna la sfera della potenzialità. Cosí:

un piccolo calcolo col quale cerco di affibiare un debito d'indulgenza, per il caso che esista, a Dio

s skromnim računom skušam dobiti odpustek od boga, če že ravno obstaja

Piovene

Era disposto a prendere il bambino in sella, purché riuscissero a mettere in piedi la madre

Pripravljen je bil vzeti otroka $\mathrm{v}$ sedlo, če se jim le posreči spraviti mater na noge

Piovene

7.3.3 Le subordinate concessive richiedono in italiano quasi esclusivamente il congiuntivo. Lo sloveno conosce in casi analoghi l'indicativo, introdotto da una congiunizone o una locuzione concessiva, oppure un ottativo il quale, però, di certo non esprime un desiderio; è stato trascinato nella nozione concessiva, cosi in italiano, come in sloveno, dalle proposizioni contenutistica. mente indipendenti, del tipo vuoi ... vuoi, sia ... sia.

... ma anche da quella delle sue bruttezze, poche o molte che siano pa $n a j$ je tega veliko ali malo

Moravia duto

Tra loro non si danneggiano, si proteggono, qualsiasi cosa sia acca-

naj se agodi karkoli

Tobino

Io non ti abbandonerò mai Bube, qualunque cosa succeda ... a qualunque pena ti condannino

Nikoli te ne bom zapustila, Bube, pa naj se zgodi karkoli, naj

te obsodijo na kakršnokoli kazen

Cassola

nonostante il barista dicesse del piacere che aveva il bar

ne glede na to, da je natakar govoril $\mathrm{v}$ kakšno čast....

Sciascia 
per bujo che sia, non è piú notte ceprav je še tema, noči ni več

Pratolini

Per quanto sia oppressa, è un dovere

Naj je še tako na tleh, je to vendarle dolžnost

Pratolini

sebbene spieghi in parte alcune contraddizioni di esso čeprav deloma pojasnjuje nekatera njegova protisiovja

Moravia

Qualsiasi cosa faccia o dica sono tutto quanto in quello che dico o faccio

Karkoli že rečem ali storim, vedno sem ves v tistem, kar sem rekel ali storil

Moravia

Pareva nato per strappare una ragazza a chiunque, sia che ridesse - le piantasse gli occhi addosso

Zdelo se je, da je rojen za to, da komurkoli prevzame dekleta, pa naj si bo samo s svojim smehom ali, če se ozre za njo

Pavese

Quel nome, o ingiuria che fosse

To ime ali žaljivka, kakorkoli je že bilo . Sciascia

gli scodellava $\mathrm{i}$ figlioli che poi, avesse il sangue guasto o che, non sopravvivevano

... menda je imela zanič kri ali kaj

Pratolini

e Amelia rideva perché nuda o vestita che sia, la modella interessa agli uomini

in Amelija se je smejala, ker ženski model, bodisi gol ali oblečen, zanima moške

Pavese

8. Vanno inclusi nella sfera potenziale anche i passi in cui viene espressa la posteriorità di un'azione. Chi riporta, in italiano, un tale fatto si mette evidentemente a un punto nel passato, al punto dell'azione della principale: nella subordinata l'azione che è posteriore è con questo già segnata come eventualmente realizzabile, potenziale, cioè. Nella concordanza dei tempi, la forma verbale usata è il condizionale, nelle subordinate temporali, invece, appare regolarmente il congiuntivo. In sloveno, il parlante non ha una tale visione dei fatti: può concordare con una principale nel passato un futuro nella subordinata, non conosce, cioè, una rigida concordanza dei tempi, e analogamente ricorre al futuro, o al presente di un verbo momentaneo, per esprimere la posteriorità in una subordinata temporale. Le subordinate temporali hanno in sloveno, tutte, delle congiunzioni (dokler, dokler ne, preden) che per sé stesse indicano la posteriorità dell'azione, vale a dire, la non-realtà; tuttavia, il condizionale, forma verbale che di per sé esprime azioni potenziali, può apparire.

Con la scena al presente, il futuro è la forma verbale piú frequente (o il presente d'un verbo momentaneo):

Non aspettate che vi anticipi ne pričakujte, da vam bom naprej izplačal Pratolini

Speriamo che non torni in sé prima che abbia finito Upajmo, da se ne bo prej osvestil, preden končam

Pratolini 
Le stesse due forme sono abituali, quando, per il parlante sloveno, la subordinata esprime, sempre dal punto del parlante, un vero futuro:

Avevo deciso di non parlare a mia moglie del racconto finché non avessi terminato di copiarlo

Odločil sem se bil, da ženi ne bom omenil zgodbe, dokler je ne pretipkam

Moravia

Decisi d'aspettare che in agosto i due tornassero neta

Odločil sem se, da bom počakal, da se $v$ avgustu onadva vrPavese

Non si trova, invece, il futuro in sloveno quando le due azioni, benché certamente in rapporto di posteriorità, sono considerate come appartenenti. al passato. Cosí:

Avevano ritirato i biglietti all'ingresso di via della Missione un'ora prima che la seduta cominciasse

Vstopnice sta dvignila na začetku Misijonske ulice, uro preden se je začelo zasedanje

Sciascia

Prima che sinistra, centro e destra si rapprendessero ... ci volle un po' di tempo

Precej časa je preteklo, preden sta lahko vskladila levico, center in desnico

Sciascia

Avevo preso l'abitudine . . . di consegnare la posta in arrivo, prima che passasse censura ...

se je bil navadil izročati ... došlo pošto naskrivaj, preden je šla $\vee$ cenzuro

Levi

dà un grido ... prima che la cinghia l'abbia toccato že preden ga je jermen dotaknil

Calvino

vuole essere di ritorno prima che lui si alzi

Marija hiti, kajti hotela bi biti doma prej, preden vstane

Pratolini

Le proibirebbe di lasciare il letto finché Ugo non fosse uscito $\mathrm{Ne}$ dovoli ji namreč vstati, preden Ugo ne odide

Pratolini

Il condizionale, certo, sottolinea la potenzialità dell'azione piú chiaramente che non l'indicativo; cosí troviamo:

Le propose di uscire appena avessero cenato

Predlagal ji je, da bi takoj po večerji šla ven Pratolini

Sono però rari i passi col condizionale in sloveno; questa forma verbale è davvero un modo, non è un tempo. Perciò è comprensibile che neanche in un apparente (per l'impiego delle forme verbali) periodo ipotetico dove in realtà troviamo una subordinata oggettiva, accompagnata da una temporale, lo sloveno ricorra al condizionale, ma piuttosto al futuro o al presente, cosí ad es.:

Ci fu chi pensò che i signori superiori dopo che il pettegolezzo si fosse fatto carne, sarebbero stati costretti a provvedere

Nekateri so mislili, da bodo morali višji gospodje kaj ukreniti, ko se je čenča utelesila Tobino 
9. La nostra indagine è stata rivolta al congiuntivo italiano e alla traduzione di questa forma verbale in sloveno. Interessava soprattutto constatare i mezzi linguistici di cui dispone lo sloveno per rendere le nozioni affidate in italiano a una speciale forma verbale, sconosciuta allo sloveno. E' peró inevitabile che ogni classificazione dei valori tenda a giustificare l'uso della forma neila prima lingua, nella lingua dalla quale si traduce.

Rispondendo ai quesiti posti allinzio della nostra analisi s può dire che lo sloveno ricorre, soprattutto, all'indicativo per rendere quello che esprime il congiuntivo italiano, affidando il compito di esprimere il desiderio, l'intenzione, il dubbio, lo stato d'animo, il giudizio, la potenzialità, ecc., a un elemento lessicale; al posto dell'indicativo possono comparire anche altre forme verbali quali il condizionale o l'ottativo.

Nella sfera volitiva tale elemento lessicale è sempre nel verbo della proposizione reggente; nella subordinata, invece, manca un qualsiasi elemento lessicale. Il condizionale e l'ottativo appaiono relativamente frequenti, nelle indipendenti troviamo anche l'imperativo. Lindicativo resta la forma verbale predominante.

Nella sfera del giudizio personale troviamo l'indicativo nella stragrande maggioranza. Se l'ottativo, ovviamente, non compare, è invece rilevante il numero dei casi con il condizionale; questa forma viene però usata soprattutto quando si esprime uno stato d'animo.

Il condizionale è molto più frequente nella sfera della potenzialità. Lo sloveno usa anche l'indicativo; il condizionale, tuttavia, è l'unica forma possibile nelle proposizioni formalmente relative, per lo piú attributive, negative; lo è nelle modali comparative, in quelle di sottrazione e in una cospicua parte delle subordinate appartenenti al nesso causale: nelle subordinate causali negative e nelle potenziali e irreali del periodo ipotetico. Le concessive, di regola, non hanno il congiuntivo, la concessività viene espressa con l'ottativo.

Quando l'italiano nel complesso della concordanza dei tempi, a causa della modalità, dà la precedenza al congiuntivo a danno del condizionale (semplice o composto che sia), lo sloveno, che non conosce la concordanza dei tempi alla latina, non cambia, vale a dire, non ricorre al condizionale per esprimere un'azione futura nel passato.

Possiamo perciò constatare che l'impiego del condizionale è il piú frequente nella sfera della potenzialità, e il piú debole nella sfera del giudizio personale - vale a dire nella sfera dove nell'italiano parlato comincia a predominare l'indicativo a spese del congiuntivo.

La genericità è espressa bene nelle due lingue: se l'italiano usa il congiuntivo, lo sloveno ricorre soprattutto al condizionale, in piú, in ambedue le lingue appare un elemento generalizzante. La stessa nozione può dunque essere espressa con mezzi diversi, ma in certe sfere la concordanza può essere completa e costante. 


\section{PREVAJANJE KONJUNKTIVA V SODOBNIH ITALIJANSKIH TEKSTIH $\checkmark$ SLOVENŠC̆INO}

Kontrastivna gramatika si je zadala za cilj primerjati jezikovne pojave $v$ dveh ali več jezikih, ne glede na njih sorodnost. Naša raziskava želi ugotoviti, kako in $s$ lakšnimi jezikovnimi sredstvi izraža slovenščina v odvisnem stavku željo, namero, ukaz, dvom, osebno sodbo in splošno potencialnost, kar je $\mathrm{v}$ italijanščini, in $\mathrm{v}$ romanskih jezikih nasploh, izraženo $s$ posebno glagolsko obliko, imenovano konjunktiv.

Avtor predvsem ugotavlja, da je uporaba konjunktiva $\mathrm{v}$ teh literarnih tekstih skoraj docela skladna $\mathrm{z}$ normo, ki jo postavljajo normativne slovnice. Avtor misli, ca je mogoče rabo konjuktiva $\mathrm{v}$ italijanščini zajeti $\mathrm{v}$ tri velike razdelke: $\mathrm{v}$ želelno sfero, $\mathrm{v}$ sfero osebne sodbe in $\mathrm{v}$ sfero potencialnosti. V želelni sferi (postavimo za primer Io leggevo subito... le cartoline...., perchè le mandasse alla questura Razgleanice sem prebral takoj ... da jih pošlje na kvesturo, Carlo Levi) in v sferi osebne sodbe (Non metterti in testa che tutti gli sbirri siano tanto stupidi Ne vtepaj si $v$ glavo, da so vsi biriči bedaki, Leonardo Sciascia) izraža italijanščina negotovost realizacije dvojno, namreč $s$ semantičnim elementom $v$ glagolu nadrednega stavka in $z$ morfosintaktičnim $\mathrm{v}$ odvisniku, se pravi $\mathrm{s}$ konjunktivom. $\mathrm{S}$ tega viđika je slovenščina za ta jezikovni pojav, za izražanje virtualnosti dejanja, $\mathrm{v}$ primerjavi $\mathrm{z}$ italijanščino revnejša, oziroma je italijanščina $v$ primerjavi s slovenščino redundantna. Razlika med jezikoma je občutna.

Manjša je razlika v potencialni sferi. Oblika, ki v slovenščini največkrat zavzame mesto italijanskega konjunktiva je pogojnik (cerchiamo... qualcosa che rompa o svari la giornata, nekaj, da bi bil dan zanimivejši in pestrejši, Cesare Pavese). Kadar se izraža splošnost ali pa je dejanje zanikano (e non più nessuno in particolare che. gli occupi il cuore in ni nikogar več, $k i$ bi imel prostor $v$ tvojem srcu, Elio Vittorini) se jezika skladata še $\mathrm{v}$ nečem: oba poznata odnosnico $\mathrm{v}$ glavnem stavku.

Slovenščina ima različne možnosti, da virtualnost dejanja na kak način izrazi, in sicer $\mathrm{z}$ glagolsko obliko, $\mathrm{ki} \mathrm{ni}$ indikativ, torej $\mathrm{s}$ kondicionalom, $\mathrm{z}$ imperativom ali $\mathrm{z}$ optativom (ta dva samo $v$ želelni sferi, optativ tudi $v$ dopustnih odvisnikih). Zdi se pomembna ugotovitev, da je kondicionala $\mathrm{v}$ sferi osebne sodbe malo, (seveda pa je virtualnost nakazana $\mathrm{v}$ glagolu glavnega stavka), torej $\mathrm{v}$ tisti sferi, kjer $\mathrm{v}$ pogovorni italijanščini uporaba konjunktiva najbolj popušča, kjer govoreči nemara občuti konjunktiv kot nekaj redundantnega (Credo che basta - iz pogovorme italijanščine - Mislim, da je dovolj). Seveda je mogoče v slovenščini izraziti virtualnost dejanja ali procesa ali stanja s kakim semantičnim elementom ob indikativu: morda, verjetno, lahko. Ce torej izločimo primere $z$ indikativom, je najbolj pogostno prevajanje italijanskega konjunktiva $\mathrm{s}$ kondicionalom, $v$ nekaterih odvisnikih pa je konđicional sploh edino mogoče jezikovno sredstvo, tako $\mathrm{v}$ modalnih stavkih irealne vsebine, $\mathrm{v}$ vzročnih odvisnikih, kadar se izraža zanikan vzrok, v potencialnem in irrealnem tipu hipotetičnih period.

Slovenščina ne pozna kondicionala $v$ stavkih, kjer se izraža zadobnost ali preddobnost $\mathrm{v}$ časovnih stavkih tipa Aveva preso l'abitudine di consegnare la posta in arrivo, prima che passasse censura se je bil navadil izročati... došlo pošto na skri$v a j$, preden je šla $v$ cenzuro, Carlo Levi: slovenščina ne izraža plastično dveh slojev $\mathrm{v}$ preteklosti (ali tudi $v$ prihodnosti), to je pridržano vezniku.

Kar zadeva prevode $\mathrm{v}$ slovenščino: priznavamo prevajalcu pravico, da strukturo $\mathrm{v}$ izvirniku obrne popolnoma po svoje, da le izrazi tisto, kar je avtor hotel povedati (gl. primer $\mathrm{v}$ par. 3). Vendar pa ugotavljamo pri vseh prevodih brez izjeme, da se prevajalci $\mathrm{v}$ poglavje o konjunktivu $\mathrm{v}$ italijanski morfosintaksi niso poglobili, saj so prevodi večkrat netočni, naj̉ bo že vzrok malomarnost ali nëznanje. Slab prevod pa seveda onemogoča primerjavo, in s tem tudi analizo. 